\title{
Internal bremsstrahlung in neutralino annihilation: revised impact on indirect detection through gamma-rays
}

\author{
M. Cannoni ${ }^{\star a}$, M. E. Gómez ${ }^{a}$, M. A. Sánchez-Conde ${ }^{b, c}$, F. Prada $^{d}$ and O. Panella ${ }^{e}$ \\ ${ }^{a}$ Departamento de Física Aplicada, Facultad de Ciencias Experimentales, Universidad de \\ Huelva, 21071 Huelva, Spain \\ ${ }^{b}$ Instituto de Astrofisica de Canarias (IAC), E-38200 La Laguna, Tenerife, Spain \\ ${ }^{c}$ Departamento de Astrofísica, Universidad de La Laguna (ULL), E-38205 La Laguna, Tenerife, \\ Spain \\ ${ }^{d}$ Instituto de Astrofisica de Andalucía (CSIC), E-18008, Granada, Spain \\ e Istituto Nazionale di Fisica Nucleare, Sezione di Perugia, Via Alessandro Pascoli, 06129, \\ Perugia, Italy \\ E-mail: mirco.cannonildfa.uhu.es
}

\begin{abstract}
We revise the impact of internal bremsstrahlung photons in the context of the constrained minimal supersymmetric standard model on $\gamma$-ray dark matter annihilation searches. As an example, we review the $\gamma$-ray dark matter detection prospects from Draco dwarf spheroidal galaxy with the MAGIC stereoscopic system and the CTA project. We find that, for a typical energy threshold of $100 \mathrm{GeV}$, the flux of high energy photons is enhanced by an order of magnitude in the stau co-annihilation region, where the signal remains still at least three orders of magnitude below the sensitivity of the instruments. However, the more optimistic scenarios for detection are found in the funnel and focus point regions of the parameter space where the effect of internal bremsstrahlung is negligible or small.
\end{abstract}

Identification of Dark Matter 2010

July 26 - 302010

University of Montpellier 2, Montpellier, France

*Speaker. 


\section{1. $\gamma$-rays from neutralino annihilation}

The detection of $\gamma$-rays coming from the annihilation of dark matter (DM) particles that should form the halo of galaxies is at present a very active field of research, that can complement other indirect and direct DM searches. Weakly interacting massive particles (WIMP) are very popular DM candidates. In particular, the relic density of the lightest neutralino of the minimal supersymmetric (SUSY) extension of the standard model (MSSM) can account for the DM amount required to explain WMAP data [1]. In the scenarios under consideration, there are three mechanisms producing photons in neutralino annihilation: (1) hadronisation and decay of the annihilation products [2] (mostly neutral pion's) provide secondary photons which show a continuous energy spectrum decreasing towards $m_{\chi}$, the maximum energy available. Typically this is the largest contribution over a wide portion of the parameter space; (2) at the one loop level neutralinos annihilate into photons through the processes [3] $\chi \chi \rightarrow \gamma \gamma$ and $\chi \chi \rightarrow Z \gamma$. Being neutralino highly non relativistic, annihilation is almost at rest, thus outgoing photons are almost monochromatic (lines) with energies $E_{\gamma} \sim m_{\chi}$ and $E_{\gamma} \sim m_{\chi}-m_{Z}^{2} / 4 m_{\chi}$, respectively. Though this gammas would give a very clear signal, the cross section is $\mathscr{O}\left(\alpha^{4}\right)$; (3) finally, internal bremsstrahlung (IB) [4] which consists on the emission of additional photons from neutralino pair annihilation into charged particles: $\chi \chi \rightarrow X \bar{X} \gamma, X$ being a charged lepton or a $W$ boson. In the Feynman diagrams, these photons can be either attached to the final state charged particles or to the virtual charged sparticle exchanged by neutralinos in $t$-channel: the latter diagrams explain the hard photon spectrum of IB near $m_{\chi}$. The cross section is $\mathscr{O}\left(\alpha^{3}\right)$, thus in principle intermediate between the two previous contribution, but as we will see, strongly dependent on the SUSY mass spectrum and couplings.

The expected flux of photons with energy above an energy threshold $E_{t h}$ set by experiments is given by

$$
F\left(E_{\gamma}>E_{\mathrm{th}}\right)=J(\Psi) \times f_{\text {susy }}\left(E_{\gamma}>E_{\mathrm{th}}\right) .
$$

Here $J(\Psi)$ is the astrophysical factor (we discuss it in the next Section) and $f_{\text {susy }}\left(E_{\gamma}>E_{\text {th }}\right.$ ) is the particle physics factor that includes all the particle physics informations. Given the three photon sources discussed above, we can write $f_{\text {susy }}=f_{\text {sec }}+f_{\text {lines }}+f_{I B}$, see Refs. [5, 6] for details.

For the numerical computation of IB effects we use DarkSusy 5.0.5 [7]. In the context of the constrained minimal supersymmetric standard model (CMSSM) the theory at the weak scale is determined by four parameters assigned at the gauge unification scale: the common scalar mass $m_{0}$, the gaugino mass $m_{1 / 2}$, the trilinear couplings $A_{0}$, the ratio of the Higgs vacuum expectation values, $\tan \beta$ and the sign of $\mu$, the Higgs mixing term, that we take positive. We require the neutralino relic abundance to be inside the cosmologically favoured interval $0.09<\Omega_{\chi} h^{2}<0.13$ (the most recent WMAP [9] interval at $3 \sigma$ is $0.094<\Omega_{D M} h^{2}<0.128$ ). We further require that SUSY models satisfy the LEP bounds on Higgs and chargino masses, $m_{h}>114 \mathrm{GeV}$ and $m_{\chi^{+}}>103.5 \mathrm{GeV}$, and constraints from $b \rightarrow s \gamma$. After the imposition of the phenomenological constraints only few regions of the parameter space survive. In these regions the relative weight of the three contributions to $f_{\text {susy }}$ and to the flux changes drastically. To illustrate this point [5] we select from our scan of the parameter space four points found in Table 1 where also the total annihilation cross section and the distinct contributions to $f_{\text {susy }}$ integrating the number of photons above $E_{t h}=100 \mathrm{GeV}$ can be read. 


\begin{tabular}{cccccccccc}
\hline Model & $\tan \beta$ & $m_{0}$ & $m_{1 / 2}$ & $A_{0}$ & $\langle\sigma v\rangle$ & $f_{\text {sec }}$ & $f_{\text {lines }}$ & $f_{I B}$ & $f_{\text {susy }}$ \\
\hline$(A)$ & 18 & 127 & 459 & -135 & 29 & 0.008 & 0.018 & 0.079 & 0.1 \\
$(B)$ & 52 & 982 & 1377 & 725 & 2600 & 0.72 & $10^{-5}$ & $10^{-5}$ & 0.72 \\
$(C)$ & 17 & 2200 & 430 & 805 & 2225 & 0.04 & 0.06 & 0.02 & 0.12 \\
$(D)$ & 51 & 8940 & 2218 & -4221 & 1203 & 0.3 & 0.003 & 0.017 & 0.32 \\
\hline
\end{tabular}

Table 1: The values of $m_{0}, m_{1 / 2}, A_{0}, m_{\tilde{\chi}}$ are in $\mathrm{GeV}$, the sign of $\mu$ is positive. $\langle\sigma v\rangle$ is given in units of $10^{-29}$ $\mathrm{cm}^{3} \mathrm{~s}^{-1}$, the $f$ 's are given in units of $10^{-32} \mathrm{GeV}^{-2} \mathrm{~cm}^{3} \mathrm{~s}^{-1}$.

Point $(A)$ is on the stau co-annihilation region of the CMSSM parameter space: the mass of the lightest stau is very close to $m_{\chi}$. Neutralino pair annihilation in $\tau^{+} \tau^{-}$mediated by $t$-channel exchange of stau has the highest annihilation cross section. Here $f_{I B}$ is the dominant contribution being 10 and 4.4 times greater than $f_{\text {sec }}$ and $f_{\text {lines }}$.

Point $(B)$ is on the funnel or resonances region: the mass of the CP-odd neutral Higgs is $m_{A} \simeq 2 m_{\chi}^{0}$ : pair annihilation into $b \bar{b}$ through $s$-channel exchange of heavy neutral Higgs bosons is the dominant channel. In this case no photon line can be attached to the virtual particles in $t$ channel exchange and the IB yield is negligible. $f_{\text {sec }}$ is the only relevant contribution.

Point $(C)$ is on the focus point or hyperbolic branch region. The mass of the lightest chargino is not much bigger than $m_{\chi}$ and neutralino pairs annihilate into $W^{+} W^{-}$through $t$-channel chargino exchange. The IB yield is small because $m_{\chi}$ is not much greater than $m_{W}$ and photons energy has a cut off which corresponds to the kinematic endpoint $x=1-m_{W}^{2} / m_{\chi}^{2}$. Here $f_{\text {lines }}$ is bigger than $f_{s e c}$ and $f_{I B}$, and the three contribution are of the same order.

Point $(D)$ is another example in the focus point region. The mass of the lightest chargino is almost degenerate with $m_{\chi}$ Neutralino pairs annihilate into $W^{+} W^{-}$through $t$-channel chargino exchange as in $C$ but in this case $m_{\chi} \gg m_{W}$ thus IB photons contribution is more important and have endpoint at the neutralino mass: here $f_{\text {susy }}$ is dominated by $f_{\text {sec }}$ even if $f_{I B}$ is greater than $f_{\text {lines }}$. Note that although $f_{I B}$ can be dominant, point $(A)$, or bigger or of the same order of $f_{\text {lines }}$, points $(D)$ and $(C)$, the bigger $f_{\text {susy }}$ is reached in point $(B)$ where $f_{I B}$ is irrelevant, thus from the point of view of the most promising scenario for detection there is no improvement: we discuss this point in a more general way in Section 3.

\section{Astrophysical target: Draco dwarf galaxy}

Gamma-rays are detected by imaging air Cherenkov telescopes (IACT) like MAGIC, HESS, VERITAS or satellites-based experiments like the Fermi satellite. For these experiments, dwarf spheroidal (dSph) galaxies around the Milky Way represent a good alternative target option respect other objectives like the Galactic Centre. DSph's are DM dominated systems with inferred very high mass-to-light ratios, and most of them are expected to be free from any other astrophysical source that might contribute to a possible $\gamma$-ray signal. Therefore, the detection of $\gamma$-rays from them would probably imply a successful DM annihilation detection. Here we consider Draco, which is one of the most studied dSph [11]. Located at $80 \mathrm{kpc}$, Draco is one of the dwarfs with more observational constraints, which helped to better determine its DM density profile. Draco has 
already been observed by some of the quoted experiments. The MAGIC telescope [12], found no gamma signal above an energy threshold of $140 \mathrm{GeV}$. As a consequence, an upper limit for the flux ( $2 \sigma$ level) was set to be $1.1 \times 10^{-11}$ photons $\mathrm{cm}^{-2} \mathrm{~s}^{-1}$, assuming a power-law with spectral index -1.5 and a point-like source. This upper limit is $\mathscr{O}\left(10^{3}-10^{9}\right)$ above the values predicted by those SUSY models used in their analysis and therefore no constraints could be put on the parameter space. Fermi collaboration reported their upper limits for a possible $\gamma$-ray annihilation signal from Draco at lower energies [13], no significant gamma emission above $100 \mathrm{MeV}$ was reported. The VERITAS collaboration [14] published results from 18.38 hours of observation of Draco finding no signal. An upper limit at $95 \%$ c.l. on the total flux was set to be $0.49 \times 10^{-12}$ photons cm $\mathrm{cm}^{-2}$ above $200 \mathrm{GeV}$. The astrophysical factor $J(\Psi)$ represents the integral of the square of the dark matter density $\rho_{D M}$ along the direction of observation $\Psi$ relative to the centre of the DM halo. For Draco, we use the cuspy DM density profile given in Refs. [6]-[5] also used by the MAGIC collaboration in their analysis [12]. In particular, we use here the value of $J(\Psi)$ integrated over the whole spatial extent of the source as the value of the astrophysical factor. This value can be well approximated by $\bar{J}=\frac{1}{4 \pi D^{2}} \int_{V} \rho_{D M}^{2}(r) d V$, with $D$ the distance from the Earth to the centre of the $\mathrm{DM}$ halo and $r$ measures distance inside the halo from the centre. In the case of Draco we take $\bar{J}=3.7 \times 10^{17} \mathrm{Gev}^{2} \mathrm{~cm}^{-5}$. We remark that in literature others models for the halo profiles are used providing slightly different values for $J$ : in particular both Fermi and VERITAS analysis assume a two parameters Navarro-Frank-White density profile [15]. The uncertainties on the astrophysical factor are of order of a factor 2-3 using different models: this uncertainty is however irrelevant with the present experimental sensitivity as we show in the next section.

\section{Results}

Armed with the previous ingredients we perform a scan on the parameter space determining $f_{\text {susy }}$ as a function of the energy threshold, which is the important quantity determining the flux once the astrophysical factor is fixed. We set $A_{0}=0$ and take two values of $\tan \beta, 10$ and 50 varying $m_{0}$ and $m_{1 / 2}$ such that the experimental constraints discussed in Section 1 are satisfied. For each value of $\tan \beta$ we separate the models with $m_{0}>2 \mathrm{TeV}$ and $m_{0}<2 \mathrm{TeV}$ in order to separate the focus point region from the stau co-annihilation and the Higgs funnel ones. In Fig. 1 the shaded areas correspond to the total $f_{\text {susy }}$, the areas inside the dot-dashed lines correspond to $f_{\text {sec }}$ and the areas inside the dashed lines give $f_{\text {lines }}$.

In panel $(a)$ of Fig. 1, where points are in the stau co-annihilation region, we can appreciate the largest contribution of IB, as shown by the point $(A)$. The absence of IB photons in the point $(B)$ is evidenced by the panel $(b)$ where points are mostly in the funnel region, while the panels $(c)$ and $(d)$ have points mostly in the hyperbolic branch and share properties with the points $(C)$ and $(D)$. To compare with the future experimental sensitivities we plot in these figures the sensitivity curves of the MAGIC telescopes in stereoscopic mode [16] and of the CTA project [17] for Draco. These curves are obtained dividing the Montecarlo simulated sensitivity for the flux (50 hours of observation time and a $5 \sigma$ detection level) by the total astrophysical factor discussed in Section 2.

Let us focus at $E_{t h}=100 \mathrm{GeV}$, the typical energy threshold for the IACT. The IB contribution is seen to be important only in panel $(a)$ corresponding to the stau co-annihilation region, as expected. The order of magnitude effect has the net result of pushing $f_{\text {susy }}$ to the value $\simeq 10^{-33} \mathrm{GeV}^{-2} \mathrm{~cm}^{3}$ 

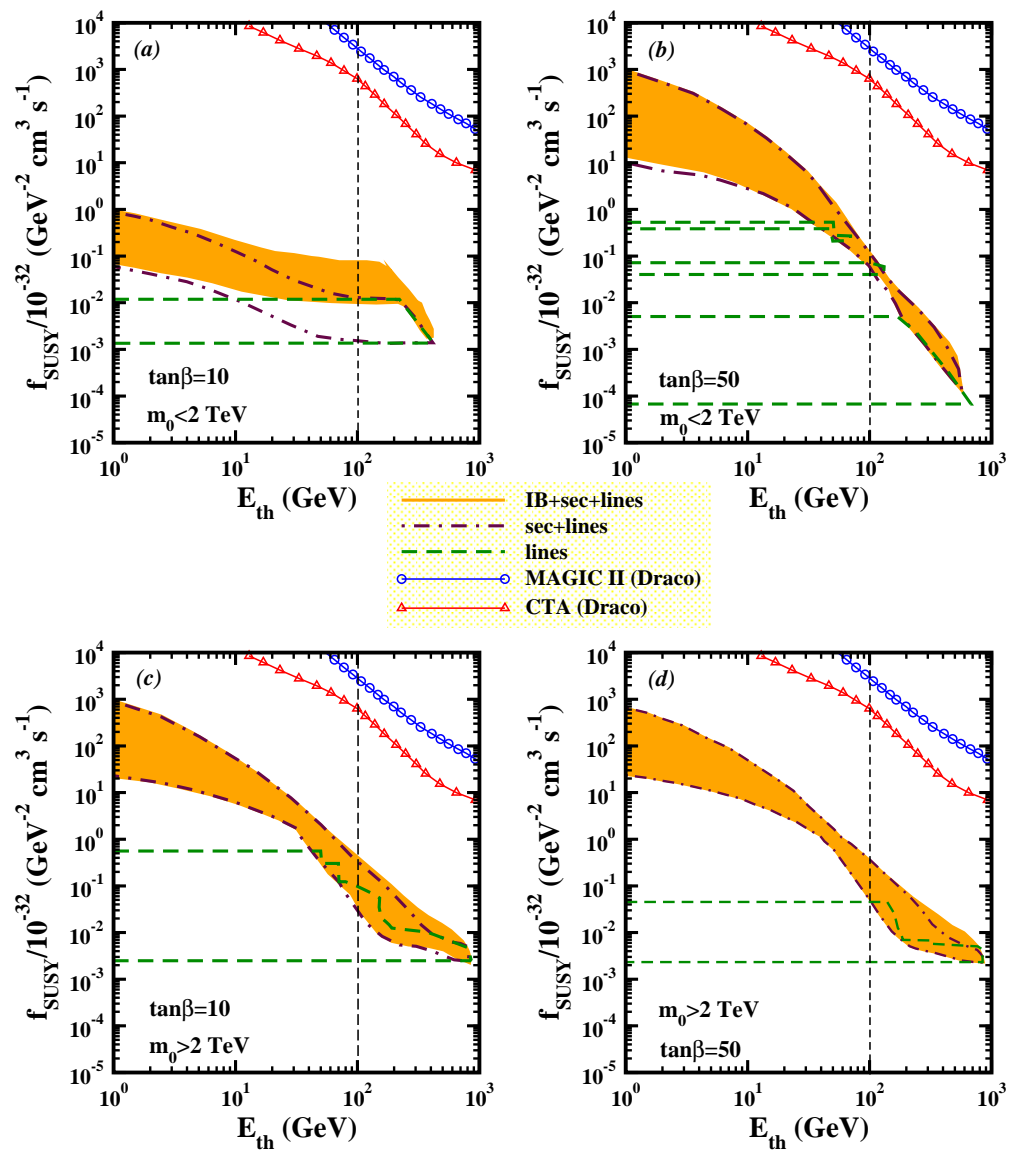

Figure 1: The particle physics factor $f_{\text {susy }}$ versus $E_{t h}$, the energy threshold of the detector. We set $A_{0}=0$ and $\mu$ positive while $m_{0}$ and $m_{1 / 2}$ have values such that the CMSSM point predicts the neutralino relic density inside the WMAP bounds satisfying all the phenomenological constraints. Also plotted are the predicted sensitivity lines of MAGIC II and CTA for Draco corresponding to 50 hours of observation time and a $5 \sigma$ detection level. The black vertical line corresponds to the typical threshold for the IACT. The abbreviations "sec" and "lines" stand for secondary and monochromatic photons, respectively. The greendashed line thus indicates the contribution of the monochromatic photons alone, the dashed-dot line the sum the monochromatic and continuous secondary photons, the orange region includes also the IB contribution.

$\mathrm{s}^{-1}$ as in panel $(b)$. However, the resulting value is smaller than the ones in panel $(c)$ and $(d)$, $f_{\text {susy }} \simeq 5 \times 10^{-33} \mathrm{GeV}^{-2} \mathrm{~cm}^{3} \mathrm{~s}^{-1}$ that is the most optimistic value. Thus we see that while IB increases by an order of magnitude the flux in the stau coannihilation region, that, anyway, is the less promising parameter space region for observation since the sensitivity of the experiments is more than three orders of magnitude above the predicted flux. Note that the same is true at a low thresholds, say $1 \mathrm{GeV}$ of interest for the Fermi satellite, $f_{\text {susy }}$ in panel $(a)$ is three orders of magnitude smaller than in panels $(b),(c),(d)$.

\section{Summary}

Although IB can be significant in the evaluation of the high energy photon flux from neutralino 
annihilation, we find that its contribution is relevant only in the stau co-annihilation region of the CMSSM parameter space. However, in this framework, the most optimistic particle physics scenarios for DM detection typically correspond to points of the parameter space where most of the flux is arises from secondary photons that do not receive an important contribution from IB. As an example of the impact of the IB on DM search, we included it in a revision of the DM detection prospects at Draco dwarf galaxy with MAGIC II and future CTA telescopes and found that at $E_{t h}=100 \mathrm{GeV}$, the predicted fluxes are still at least three orders of magnitude below the sensitivity of the IACTs.

\section{Acknowledgments}

M. C. is a MultiDark fellow. The authors acknowledge the MultiDark project of Spanish MICINN Consolider-Ingenio: CSD2009-00064, the project P07FQM02962 funded by Junta de Andalucia, and the Spanish MICINN-INFN(PG21) projects FPA2009-10773, FPA2008-04063-E.

\section{References}

[1] G. Jungman, M. Kamionkowski and K. Griest, Supersymmetric dark matter, Phys. Rept. 267, 195 (1996) [arXiv:hep-ph/9506380]; G. Bertone, D. Hooper and J. Silk, Particle dark matter: Evidence, candidates and constraints, Phys. Rept. 405, 279 (2005) [arXiv:hep-ph/0404175].

[2] J. Silk and M. Srednicki, Cosmic-ray antiprotons as a probe of a photino-dominated universe, Phys. Rev. Lett. 53, 624 (1984); J. E. Gunn, B. W. Lee, I. Lerche, D. N. Schramm and G. Steigman, Some astrophysical consequences of the existence of a heavy stable neutral lepton, Astrophys. J. 223, 1015 (1978); F. W. Stecker, The Cosmic Gamma-Ray Background From The Annihilation Of Primordial Stable Neutral Heavy Leptons, Astrophys. J. 223, 1032 (1978); Y. B. Zeldovich, A. A. Klypin, M. Y. Khlopov and V. M. Chechetkin, Astrophysical Constraints On The Mass Of Heavy Stable Neutral Leptons, Sov. J. Nucl. Phys. 31, 664 (1980) [Yad. Fiz. 31, 1286 (1980)]; J. A. R. Cembranos, A. de la Cruz-Dombriz, A. Dobado, R. A. Lineros and A. L. Maroto, Photon spectra from WIMP annihilation, arXiv:1009.4936 [hep-ph].

[3] L. Bergstrom and P. Ullio, Full one-loop calculation of neutralino annihilation into two photons, Nucl. Phys. B 504, 27 (1997) [arXiv:hep-ph/9706232]; Z. Bern, P. Gondolo and M. Perelstein, Neutralino annihilation into two photons, Phys. Lett. B 411, 86 (1997) [arXiv:hep-ph/9706538]; P. Ullio and L. Bergstrom, Neutralino annihilation into a photon and a Z boson, Phys. Rev. D 57, 1962 (1998) [arXiv:hep-ph/9707333].

[4] L. Bergstrom, Radiative process in dark matter photino annihilation, Phys. Lett. B 225, 372 (1989);

R. Flores, K. A. Olive and S. Rudaz, Radiative processes in LSP annihilation, Phys. Lett. B 232, 377 (1989); L. Bergstrom, T. Bringmann, M. Eriksson and M. Gustafsson, Gamma rays from heavy neutralino dark matter, Phys. Rev. Lett. 95, 241301 (2005) [arXiv:hep-ph/0507229].

V. Barger, Y. Gao, W. Y. Keung and D. Marfatia, Generic dark matter signature for gamma-ray telescopes, Phys. Rev. D 80, 063537 (2009) [arXiv:0906.3009 [hep-ph]]; T. Bringmann, L. Bergstrom and J. Edsjo, New Gamma-Ray Contributions to Supersymmetric Dark Matter Annihilation, JHEP 0801, 049 (2008) [arXiv:0710.3169 [hep-ph]]; L. Bergstrom, T. Bringmann and J. Edsjo, Complementarity of direct dark matter detection and indirect detection through gamma-rays, arXiv:1011.4514 [hep-ph]. 
[5] M. Cannoni, M. E. Gomez, M. A. Sanchez-Conde, F. Prada and O. Panella, Impact of internal bremsstrahlung on the detection of gamma-rays from neutralinos, Phys. Rev. D 81, 107303 (2010) [arXiv:1003.5164 [astro-ph.CO]].

[6] M. A. Sanchez-Conde, F. Prada, E. L. Lokas, M. E. Gomez, R. Wojtak and M. Moles, Dark matter annihilation in Draco: New considerations of the expected gamma flux, Phys. Rev. D 76, 123509 (2007) [arXiv:astro-ph/0701426].

[7] P. Gondolo, J. Edsjo, P. Ullio, L. Bergstrom, M. Schelke and E. A. Baltz, DarkSUSY: Computing supersymmetric dark matter properties numerically, JCAP 0407, 008 (2004) [arXiv:astro-ph/0406204].

[8] K. A. Olive, Dark Matter in SuperGUT Unification Models, arXiv:1009.0232 [hep-ph].

[9] D. Larson et al., Seven-Year Wilkinson Microwave Anisotropy Probe (WMAP) Observations: Power Spectra and WMAP-Derived Parameters, arXiv:1001.4635 [astro-ph.CO].

[10] M. E. Gomez, T. Ibrahim, P. Nath and S. Skadhauge, WMAP dark matter constraints and Yukawa unification in SUGRA models with CP phases, Phys. Rev. D 72, 095008 (2005) [arXiv:hep-ph/0506243]; An improved analysis of $b->$ s gamma in supersymmetry, Phys. Rev. D ibid. 74, 015015 (2006) [arXiv:hep-ph/0601163]; M. Cannoni and O. Panella, Neutralino Dark Matter and Higgs mediated Lepton Flavor Violation in the Minimal Supersymmetric Standard Model, Phys. Rev. D 81, 036009 (2010) [arXiv:0910.3316 [hep-ph]].

[11] C. Tyler, Particle dark matter constraints from the Draco dwarf galaxy, Phys. Rev. D 66, 023509 (2002); N. W. Evans, F. Ferrer and S. Sarkar, A 'Baedecker' for the dark matter annihilation signal, Phys. Rev. D 69, 123501 (2004) [arXiv:astro-ph/0311145]; L. Bergstrom and D. Hooper, Dark matter and gamma-rays from Draco: MAGIC, GLAST and CACTUS, Phys. Rev. D 73, 063510 (2006) [arXiv:hep-ph/0512317]; S. Profumo and M. Kamionkowski, Dark matter and the CACTUS gamma-ray excess from Draco, JCAP 0603, 003 (2006) [arXiv:astro-ph/0601249]; S. Colafrancesco, S. Profumo and P. Ullio, Detecting dark matter WIMPs in the Draco dwarf: a multi-wavelength perspective, Phys. Rev. D 75, 023513 (2007) [arXiv:astro-ph/0607073]; L. E. Strigari,

S. M. Koushiappas, J. S. Bullock and M. Kaplinghat, Precise constraints on the dark matter content of Milky Way dwarf galaxies for gamma-ray experiments, Phys. Rev. D 75, 083526 (2007) [arXiv:astro-ph/0611925]. T. Bringmann, M. Doro and M. Fornasa, Dark Matter signals from Draco and Willman 1: Prospects for MAGIC II and CTA, JCAP 0901, 016 (2009)[arXiv:0809.2269 [astro-ph]].

[12] J. Albert et al. [MAGIC Collaboration], Upper limit for gamma-ray emission above $140 \mathrm{GeV}$ from the dwarf spheroidal galaxy Draco, Astrophys. J. 679, 428 (2008) [arXiv:0711.2574 [astro-ph]].

[13] A. A. Abdo et al., Observations of Milky Way Dwarf Spheroidal galaxies with the Fermi-LAT detector and constraints on Dark Matter models, Astrophys. J. 712, 147 (2010) [arXiv:1001.4531 [astro-ph.CO]].

[14] The VERITAS Collaboration, VERITAS Search for VHE Gamma-ray Emission from Dwarf Spheroidal Galaxies, Astrophys. J. 720, 1174 (2010) [arXiv:1006.5955 [astro-ph.CO]].

[15] J. F. Navarro, C. S. Frenk and S. D. M. White, A Universal Density Profile from Hierarchical Clustering, Astrophys. J. 490, 493 (1997) [arXiv:astro-ph/9611107].

[16] P. Colin et al. [MAGIC collaboration], Performance of the MAGIC telescopes in stereoscopic mode, arXiv:0907.0960 [astro-ph.IM].

[17] M. Doro [CTA collaboration], CTA - A Project for a New Generation of Cherenkov Telescopes, arXiv:0908.1410 [astro-ph.IM]. 